\title{
THE ROLE OF NON-INTRUSIVE OPERATOR LOGGING TO SUPPORT THE ANALYSIS AND GENERATION OF PRODUCT ENGINEERING DATA USING IMMERSIVE VR
}

\author{
J.M. RITCHIE ${ }^{*}$, R.G. DEWAR ${ }^{1}$, G. ROBINSON ${ }^{1}$,J.E.L SIMMONS ${ }^{1}$, F.M. NG ${ }^{2}$, \\ ${ }^{1}$ Scottish Manufacturing Institute, Heriot-Watt University, Edinburgh EH14 4AS, UK \\ ${ }^{2}$ Institute of High Performance Computing, \#02-11/12 The Rutherford, Singapore 118261 \\ *Corresponding author. Email: J.M.Ritchie@hw.ac.uk
}

Abstract:

Virtual reality (VR) has the potential to substantially alter the manner in which products of the future are engineered. Currently there are many applications of VR during the product engineering process from design and analysis through to process planning, assembly, machining and shop floor layout. VR takes a multitude of forms, including immersive, desktop, augmented, and is rapidly developing as a tool that can be used in the engineering of products.

This paper looks at the use of immersive VR as a tool for analyzing both design and manufacturing product engineering activities in a series of research projects involving assembly planning and cable harness design. It focuses particularly on the use of the non-intrusive logging of users in such environments as a means to obtaining a rich data source for activity analysis and its potential within computer integrated manufacturing environments as a means of providing downstream engineering data.

Key words: immersive virtual reality, design, manufacture, assembly, cable harness, knowledge acquisition, measurement, experimentation.

\section{Introduction}

Virtual reality (VR) is an enabling technology which is developing rapidly within the engineering sector. It has been used for a number of years as a means of evaluating engineering designs as well as improving the awareness of engineers in their perception of design and manufacturing solutions (Jayaram et al. 2001, Ritchie 2004).

For design, VR systems are changing the manner in which engineers develop products and work together to generate ideas, embody their concepts and produce the information necessary for cost effective manufacture. Gomes de Sa and Zachmann (1999) see a role for immersive VR throughout the whole product development cycle; they address both the usability issues and the importance of human factors research as part of such product engineering studies. Cruz-Niera (1998) describes a C2 CAVE environment being used for architectural design so that students could appreciate a model at full scale and mentions the importance of recording and storing some form of design intent as activities are carried out. Weyrich and Drews (1999) used a virtual workbench for design and found that the method appears to effectively support how engineers 
think during the design process. Also COVIRDS (COnceptual VIRtual Design System) demonstrated the potential interactive capabilities of immersive virtual design (Dani and Gadh 1997); using a hand tracking and voice input interface to a VR-based CAD environment where rapid concepts can be modeled through freeform shape creation, an important advance in interactive virtual design; these types of interactive system are now on the market and available for use (Digital Art Forms 2006).

Assembly planning is another product engineering task that is well suited to immersive VR. The ability to pick up objects, move them around and place them in their desired positions and orientations would appear to give an intuitive feel to the manner in which an individual can interact with the data or product. VR research applications are showing promise and the methodology of collecting data discussed in this paper demonstrate this. Early applications using immersive VR include that of Heger and Richter (1997) which allowed a process planner to be immersed in a single-user environment, choose assembly tools and snap objects into place by ergonomically moving them close to the final assembly position enabling the assembly sequence to be defined, checked and changed if necessary. The VADE (Virtual Assembly Design Environment) system developed at Washington State University allowed engineers to investigate assembly construction requirements in conjunction with functional design needs as well as highlighting the importance of integrating $\mathrm{CAD} / \mathrm{VR}$ environments so that the best-fit tool can be used for product engineering as required (Jayaram et al 1997). Gupta (1995) highlighted how, in his Virtual Environment for Design for Assembly (VEDA), the designer sees a visual representation of the objects which make up an assembly using sound cues and haptic force feedback.

Kay and Constantin (1997) highlight that, "The integration of design, manufacturing, shop floor control and management activities is the ultimate goal of CIM (Computer Integrated Manufacturing) and concurrent engineering systems."

From these limited studies it can be seen that VR, especially in its immersive form, has a role to play in product engineering. The research work highlighted in this paper demonstrates how the ability to log the user within these environments provides a capability to generate design and manufacturing information as well as evaluate the activities being carried out. Direct observation can be intrusive because subjects are aware that they are being observed and their performance measured; changes in behavior, known as the Hawthorne Effect (Preece et al. 1994) can take place that adversely affect the quality of the data being collected. For this reason, the ability to log the user without them being aware of an observer can have a benefit on the quality of data recorded as the use of an immersive virtual environment (VE) in this way will be less intrusive to the operator from whom the knowledge is being acquired.

This paper discusses the novel application and potential capability of immersive VR in product assembly planning, knowledge elicitation and product design with the support and analyses of user logging where logging is defined as recording and storing any data relating to the movements, interactions and activities of the system user automatically and non-intrusively.

\section{Generating Assembly Plans and Simple Expert Rules via User Logging}

\subsection{Virtual Assembly Planning}

The immersive VR assembly research system developed for this research (Dewar 1998) was based on a hardware platform comprising a Hewlett-Packard 725/75 workstation incorporating the HP-UX 10.01 operating system with a graphics accelerator for driving a head mounted display (HMD) and a 3D mouse. User HMD and 3D mouse tracking was carried out via a Polhemus tracker. The virtual environment was programmed using dVS/dVISE (version 3.1) which also enabled the time-phased dynamic logging of all of 
the user's activities, e.g. which models were picked up, moved, final locations and method of joining as well as menu options chosen from the floating toolboxes.

The reason for using an immersive system over desktop VR was highlighted in the early evaluation trials where desktop assembly tasks took considerably longer and users found great difficulty in navigating around and understanding their environment. The immersive VR systems offered an intuitive, unrestrained, real-time means of interaction which helped highlight clashes and inconsistencies in assemblies which cannot be easily be seen with a conventional, flat-screen CAD interface. The structure of the UVAVU (Unbelievable Vehicle for Assembling Virtual Units) assembly system is shown in figure 1 and utilized both proximity and collision snapping (Dewar et al. 1997a, Dewar et al 1997b) due to the lack of haptic feedback and more elaborate visual cues.

Figure 1 The UVAVU assembly system.

UVAVU utilized 3D CAD models and user interaction such that a time-phased data file containing sequential information was output directly relating to the manner in which the assembly is built (figure 2).

Figure 2. UVAVU's 'joining' toolbox in use during assembly planning.

Post-processing of the file generated a readable assembly plan which could then be used to build the real product. The system automatically recorded the user's inputs, positional information, assembly times and system performance data, joining methods and assembly sequence as the assembly task proceeded.

Prior to actual product assembly by expert users, the system was rigorously pilot tested and validated using a large population of mechanical engineering students building virtual and real toy assemblies to ensure that the system was user friendly, to analyze the differences between real and virtual design times, to determine if the sequences generated in the virtual world matched those in the real world and to check the system's feasibility (Dewar 1998). The main phase of experimental testing involved four assembly planners from the industrial partner company using the system to build a major mechanical assembly, generate the plans from the user log files and then ask shop floor operators to build the actual product to see if these were feasible; note that these operators had no idea how the original plans had been generated. The experts were asked to assemble a 40column printer transport unit (figure 3 and figure 4) used to deliver account statements to customers within an automated teller machine (ATM).

Figure 3. Actual 40-column printer transport unit.

Figure 4. Virtual model of 40-column printer transport unit.

Figure 5 shows the liaison chart generated for the assembly from the log file data.

Figure 5. Liaison chart for 40-column printer transport unit.

The post-processed assembly plans generated from the virtual planning tasks HTML log file outputs are shown on Table 1. The original company document assembly planning sequence is labeled 'AMCD'.

Table 1. Industrial trial top level assembly plans for the transport unit.

These were then given to four assembly operators and successfully used to build the final product. In each case, all of them were able to assemble the product successfully using all four variants of the assembly plan (figure 6). 
Figure 6. Building the product in the real world.

This study successfully demonstrated that manufacturing information derived from the non-intrusive virtual world log files was consistent with that derived from the natural world and, therefore, the information was both useful and practical in an industrial setting; the first time this had been done using immersive VR. Subassemblies and stability criteria were correctly taken into consideration by the experts as they used the system, which would be expected. For more inexperienced users more realistic Newtonian-type environments may be necessary. However, user logging had enabled both liaison chart information and practical assembly plans to be generated.

\subsection{Generating Simple Expert Rules from Log Files}

In modern companies the acquisition of knowledge is seen as more than just a means of defining information for expert system development. Many organizations now see knowledge as an asset that needs to be formalized and invested in for the benefit of the company. If this is possible then it can be reused effectively. Indeed, it is the view of Nonacka and Takeuchi (1995) that "organizational knowledge creation" was at the heart of the way in which Japanese companies innovated, leading to a significant competitive advantage in the 1970s and 1980s. The research question here was: "Can immersive VR and associated user logging be used to acquire expert knowledge of some kind and transfer tacit knowledge into formalized explicit knowledge?"

In order to answer this it was decided to follow on from the previous virtual assembly planning work and determine whether a novel knowledge elicitation methodology could be developed and tested which would enable the acquisition of expert knowledge from non-intrusive logging data. Since the VR system has control of the complete virtual assembly environment, it is possible to record and log most aspects of the subject's behavior whilst assembling a set of virtual components. In this research, assembly usage data gathered from a virtual assembly environment was combined with the definition and evaluation of various component and joint attributes to produce assembly data in a suitable form for the automatic induction of rules. The methodology's overall structure is shown in figure 7 and includes four main processes:

1. Define the salient component attributes using any typical assembly (repertory grid analysis).

2. Evaluate the attributes for a particular assembly (attribute assessment).

3. Record the expert assembly usage data for that assembly (virtual assembly environment).

4. Generate rules relating the components to the assembly methods (induction engine).

Figure 7. Overall form of the knowledge elicitation process.

The following four sections describe each of the main processes with examples given where appropriate.

\subsubsection{Define \& Evaluate Salient Component Attributes (Repertory Grid Analysis)}

The psychologist Kelly (1995) developed a hypothesis of human thinking called personal construct theory. According to this, each person is a 'scientist' with their own personal model of the world about them. The scientist classifies and categorizes their world, developing theories about it. Based on these theories, individuals are able to anticipate and then act on the basis of their anticipations. The repertory grid technique is a method for eliciting and analyzing an expert's model of a problem and is based on Kelly's idea of personal constructs.

In order to evaluate the main constructs associated with the product, four assembly planners were asked to complete a questionnaire as shown in Table 2; this related to the component types used in a similar product, namely a credit card feeder mechanism. 
Table 2. Component attribute questionnaire.

The results from the questionnaire process showing relationships between components and attributes are summarized in Table 3.

Table 3. Results from expert's questionnaire.

\subsubsection{Recording Usage Data via an Immersive Virtual Environment}

In order for the induced rules to be created it is important to know how the product was built. Therefore the detailed usage data used was that generated for the printer assembly and post-processed to produce the process plan. As an example, for the rule "heavy, costly items tend to be base parts" to be induced it is vital to know the assembly sequence. Normally, to obtain detailed usage data for an assembly, an assembly expert would need to be closely observed putting together a product. However, as has been previously discussed, a non-intrusive method of observing the user is of benefit in reducing the Hawthorne Effect (Reece et al 1994). For this reason, engineers were observed solely by means of the data recorded in the immersive virtual environment. Using the same data generated by the same four expert assembly planners as shown in Table 1 plus additional information on joint-types it was then possible to generate expert rules.

\subsubsection{Generate Expert Rules via Rule Induction}

The data gathering and knowledge elicitation techniques mentioned so far provide sufficient data to use automatic rule induction (Hart 1996). Induced rules generally take the form of IF-THEN statements which connect various logical conditions in the IF clauses with a value or values of the target variable in the THEN clause.

The implementation of this KE methodology used the freely available CN2 induction algorithm developed in the late 1980s at the Turing Institute in Glasgow, UK (Clark and Niblett 1989, Clark and Boswell 1991). This algorithm is designed for, "the efficient induction of simple, comprehensible production rules in domains where problems of poor description language and/or noise may be present."

An important consequence of this modified algorithm is that it tends to produce shorter, less complex rules that are also much easier to demonstrate and explain to subjects when seeking verification of the induced rules. Although other induction algorithms exist CN2 was also chosen for its simplicity and ease of use since the purpose of this research was to prove the principle of rule induction and not to compare different methods of achieving this.

\subsubsection{Examples of Rule Induction}

These examples again concern the 40-column printer transport and use the attribute and usage data derived in the previous two sections. By observing the usage data, it was decided to investigate an example employing the component attributes of mass and handleability. These were to be considered along with whether or not a component was grabbed and brought to another component or subassembly for subsequent joining. The target attribute grabbed can be deduced where the first component in each joint pair is the one that was picked up in the VE. Since there are twelve components and four subjects there are 48 examples in the training set in Table 4. The first twelve entries are associated with participant A, the next twelve with B, and so on. In practice, the values are simply pasted into a text file for the induction engine to read. 
Table 4. First training set for induction engine.

From this training set, $\mathrm{CN} 2$ produced the following rules:
RULE 1
IF Mass $=$ Light
AND Handleability $=$ Medium
THEN Grabbed $=$ y $\lfloor 2$ 8 $\rfloor$
RULE 2
IF Mass $=$ Light
ANI Handleability $=$ Easy
THEN Grabbed $=$ y $\lfloor 4$ 28]

The total of the two figures in square brackets represents the total number of cases in the training set, with the rightmost of the two being the number of cases that agree with the rule. Rule 1 states that light components that are 'moderately' easy to handle tend to be picked up and brought for joining. In other words they tend to be base parts. Rule 2, by virtue of being true more times in the training set, is more reliable and indicates that light components that are easy to handle tend to be picked up more often than not.

The other example investigated looked at the joint attributes as opposed to those for the components. Before assembly there are twelve rigid bodies, each with six degrees-of-freedom (DOF). It could be said that there is a total of 72 DOF in the system. Furthermore, a convention has been adopted such that after a subassembly of two or more components is created, that subassembly becomes fixed save for any internal relative freedoms between components. Therefore, if a guide and tie bar are joined by an insertion, the resulting subassembly has 1 DOF since the tie bar can still rotate about its own longitudinal axis and the subassembly as a whole is assumed by this convention to be fixed. In other words, the joint has reduced the freedoms of the components by 11 degrees and the whole system now has only 61 DOF. Extending this convention for the eleven joints that each of the four participants make, the 44 examples in Table 5 can be arrived at. The table considers the change in DOF, the type of joint involved and whether an identifiable new subassembly has been started as opposed to transient stages. For the transport mechanism, the identifiable subassemblies consist of:

(a) three guides and two tie bars, called a guide subassembly;

(b) a side frame and one or more guide subassemblies;

(c) the completed assembly itself. 
Table 5. Second training set for induction engine

The CN2 induction engine gave the following rules:
RULE 3
IF DeltaIDOF $<8.50$
ANI Jointype $=$ Insert
RULE 4
THEN NewSub $=$ n [0
24|
IF DeltaDOF $>$ 8.50
ANI JointType $=$ Insert
THEN NewSub = y [0 8]

\author{
RULE 5 \\ IF JointType = Serew \\ THEN NewSub = y IO \\ $12]$
}

$\Delta$

Rule 3 indicates that, for inserted joints, if the change in DOF is below a certain value there has been no identifiable subassembly created. Rule 4 again deals with insertion joints but shows that when the change in DOF is high enough a new subassembly has been started. Rule $\mathbf{5}$ states that screwed joints signal a new subassembly. In other words, this example indicates that joints which make components most stable tend to be carried out first. From a design and manufacturing perspective these three rules have other, quite profound implications. For inserted joints (Rules 3 and 4), if the change in degrees of freedom is kept small during the design for assembly (DFA) phase then it is more likely that a separate subassembly will not be required. This would not only lead to a more efficient overall assembly operation in terms of time, due to a reduction in manipulation, but also at least one less subassembly and associated operations - implying potentially less interoperation time. The use of screwed joints (Rule 5) for this type of product always implies a new subassembly. Not only will this take more time to carry out but the implications are that redesigning these into snap fits would substantially reduce both operation counts and associated product lead times. The component and joint-type rules induced were presented to expert assembly planners at the industrial partner who have subsequently confirmed that they do articulate the reasoning process that they adopt when they approach an assembly task. This work established a novel methodology to non-intrusively elicit expert manual assembly planning knowledge as well as demonstrating for the first time that immersive VR and associated data logging has the potential to be a useful knowledge acquisition tool and appears to suppress the Hawthorne affect.

\section{Gathering Data for VR:CAD Comparisons via User Logging}

Following the successful use of immersive VR in a manufacturing task it was decided to investigate its potential within an interactive design environment. The industrial partner in the previous research was asked what aspects of their new product development process caused major problems in trying to get new products to market. The key area highlighted was cable harness design and, after investigating the CAD functionality to produce this information in the design office, it was determined that immersive VR could be made to operate in such a way as to enhance the design of this type of product.

Figure 8. A typical cable harness assembly.

As shown in figure 8, cable harnesses comprise individual cables bundled together to provide electrical and data connections to various modules within pieces of electromechanical equipment.

Early cable harness design work was carried out in an attempt to automate cable harness routing (Conru 1994). Other work used genetic algorithms to tackle the same problem (Conru and Cutkosky 1993). Wolter and Krol (1996) routed 'strings' around 'solid' parts and in some projects robot path planning has been used and applied to piping systems as a routing solution (Zho and Latombe 1991). Studies at Iowa State University (Fischer et al 2002) developed a VR system for routing flexible hoses which validated VR as a practical tool; however, there was no attempt to analyze its effectiveness as an interactive design tool. None of this research had been used by industrial users and VR systems had not been compared to CAD equivalents.

An immersive VR cable harness design tool was developed for evaluation using industrial cable design experts which compared their performance against the same solutions generated in a variety of CAD 
environments. This cable design study involved a wide range of industrial partners who were investigated to determine a generic process for cable harness design ( $\mathrm{Ng}$ et al 2000) and was used as a basis for the design of the eventual virtual cable harness design platform (figure 9).

Figure 9. Schematic model of the cable harness product design process.

A VR-based toolset was produced as an experimental platform and concentrated on routing cables through a generic assembly representing the main sub-system of a product (figure 10).

Figure 10. The generic test assembly.

In designing the VR toolset, the engineering designers were provided with a design which represented the actual design tasks in a virtual world. The initial research system was called CHIVE (Cable Harnessing In Virtual Environments) and was implemented on the same platform as used in the virtual assembly trials. The user interacted with the data by means of a head mounted display (HMD), a 3D mouse and pop-up menus. The general algorithmic structure of the system is illustrated in the flow diagram in figure 11.

Figure 11. CHIVE system flow diagram.

The designers generated cables by creating points in 3D space which are joined together to produce cable elements and cable lengths as shown in figure 12.

Figure 12. CHIVE being used to route a cable harness.

Other cable creation tools allowed the designer to follow, or partially follow, existing cable routes, drag a cable through the assembly, scale the environment, bundle cables together and treat them as one large 'cable' and bend cables around obstacles. The system enabled the user to identify the most appropriate routing strategies and harness configurations to determine cable routes through the product and then select the relevant cable types. As this design activity was carried out the user actions were logged in a time-phased manner to measure their actual design performance, the lengths of cables being generated, the types and number of inputs from the various peripherals as well as the sequential order in which the design attributes were added (figure 13).

Figure 13. An example of the output log file from CHIVE.

Once the harness layout was complete, wiring lists were generated for the various solutions (figure 14).

Figure 14. Wiring list generated by CHIVE.

Field trials were carried out to investigate the system's effectiveness at creating cable harnesses to compare its performance with commercial CAD equivalents.

Since the number of expert cable design engineers available was small and was already a sample drawn from a small population, a variation on the "single-case experiment" was adopted (Hayes 2000) and no statistical testing was performed on the data. Five experienced cable harness CAD designers were selected from the four industrial collaborators participating in the research. Their CAD experience ranged from three to fifteen years and none of them had used an immersive VR system before. The VR system's 'expert' cable harness designer was a researcher with three years experience in using and developing the VR system. Pro/ENGINEER ${ }^{\text {TM }}$, SolidDesigner ${ }^{\mathrm{TM}}$ and CATIA ${ }^{\mathrm{TM}}$ (the piping module) were the CAD packages compared to CHIVE in this research (figure 15).

Figure 15. Various solutions on the CAD packages and CHIVE. 
By comparing task completion times (TCTs) logged on the system it was found that, for a simple single-level layout task, the participants took between two and four times longer to complete the routing task using the proprietary CAD systems (see Table 6). 
Table 6. TCT comparisons between CAD systems and CHIVE (single level routing).

Direct observations and video analysis showed that only half of the participants made errors in CHIVE compared to using CAD. Multilevel assembly routing was compared in a second set of trials; the TCT comparison depicted in the bar chart (figure 16) showed that CHIVE gave productivity gains of between 3:1 and 5:1.

Figure 16. TCT comparisons between CAD systems and CHIVE (multi-level routing).

Other measures logged on CHIVE and obtained from video analysis of the CAD systems showed that the number of mouse clicks and keyboard entries reduced dramatically on the VR system by factors of 2-5 times and point to the fact that interface issues as well as immersion may explain why productivity gains were apparent (Table 7 and Table 8).

Table 7. Other measures for CAD and VR system comparison (single-level routing).

Table 8. Other measures for CAD and VR system comparison (multi-level routing).

This work demonstrated that the TCTs obtained by the participants were between two and five times faster when using VR than current CAD systems, even with CAD bias during the trials.

The VR interface, with fewer mouse clicks and keyboard inputs, easier 3D navigation and quicker point creation had a significant impact on TCTs which indicated that participants found the VR system comparable to current commercial CAD systems. It also demonstrated that immersive VR can be used as an interactive design tool. In this work it was difficult to separate the influence of the graphical user interface of the VR system from the influence of the cable harness toolset therefore it is arguable whether the positive aspects of the VR system over the CAD systems are due to the software tools provided to the designer or the more sophisticated and intuitive graphical user interface. Therefore it was necessary to investigate how the design task was broken down to determine which aspects of such systems were being used for design per se or for support tasks, e.g. navigation, menu interaction, etc. The next stage of this research is now discussed.

\section{User Logging as a Means of Analyzing a Detailed Immersive VR Design Task}

In order to attempt to determine how these benefits were achieved, it was decided to investigate and categorize the design activities carried out by a designer using the immersive VR system via non-intrusive user logging. Also, current literature points to the fact that when using HMDs the maximum safe working time to which people can be exposed to the VE is around 20 minutes in order to reduce adverse effects (Howarth 1997). If this type of technology is to be used as a genuine design tool within engineering then an understanding must be obtained of how to maximize the effective design time of the product engineer so that they can use it effectively during appropriate creative design or planning activities. For this reason the novel design categorization system developed would also be useful to breakdown the cable harness design tasks whilst working in an immersive VE. These would provide a tool for future researchers investigating cable harness and other product engineering activities. Due to the greater complexity of the cable harness design system required in this experimentation, e.g. the addition of connectors and fasteners, more elaborate logging and more complex design tasks and models, it was decided to develop a new cable design system called COSTAR (Cable Organization System Through Alternative Reality). This was implemented on an SGI® Octane $^{\mathrm{TM}}(2 * 600 \mathrm{MHz}$ IP30 processors, $768 \mathrm{MB}$ RAM, 9GB HDD) with V12 dual head graphics (2*VPro V12 graphics boards each with $128 \mathrm{MB}$ graphics RAM) driving two SGI ${ }^{\circledR}$ F180 flat panel displays or each 
eye of a stereo head-mounted display (HMD). Peripherals attached to the system include a V8 stereo headset (Virtual Research Systems, Inc.), Flock of Birds ${ }^{\circledR}$ magnetic tracking system (Ascension Technology Corporation) and Pinch ${ }^{\circledR}$ Gloves (Fakespace Systems, Inc.). The software was developed using SENSE8® WorldToolKit ${ }^{\circledR}$ (WTK) release 9 (multiprocessor/multi-pipe version), running on IRIX (version 6.5.21) using an object-oriented approach, with the implementation being written in $\mathrm{C}++$. The system enables the user to design cable harness assemblies within the immersive VR environment, with all design functions including the creation of new objects being performed while the user is immersed. Interactions were achieved by means of a custom-built menu system and pinch gestures in addition to the spatial input afforded by the Flock of Birds system (figure 17).

Figure 17. Interacting with CO-STAR.

All menu actions, manipulations and creation of objects are logged in detail. A suite of Perl scripts were used to post-process these resultant large data files and extract a variety of performance measures including navigation statistics, menu usage, help usage, number of times a certain design tool was used, the time for which this was used for and the number of times functions were cancelled by the user.

Cables can be created as before with points being created quickly and effectively in free 3D space with cable sections joining them together to produce the actual cable form and the dragging and dropping points or sections to manipulate cables around obstacles if required; figure 19 shows the system in use.

Figure 18. Various cable design options within CO-STAR.

All of the tasks occurred in the same 'product' model (see figure 22) and involved consecutive stages of the overall cable harness design process; namely 'outline design', 'detailed design', and 'redesign'.

Fig 19 CO-STAR cable harness design assembly task

The users were given a realistic cable design problem for which they had to produce a viable solution. Although constrained so that comparisons could be carried out, there was enough flexibility within the tasks to evaluate the manner in which the system and technology supports or hinders the engineer during their creative work and the manner in which individuals produce solutions to the design problem. This ensured that there was a design problem for the system user to solve and prevented the evaluation process becoming a prescriptive button pushing exercise. The tasks developed for the experiments mirrored real tasks as observed on the industrial partners' sites.

The first task (Outline Design) was to generate two new electrical interconnections within the product model. Each of these interconnections had to join two specific connectors within the model and have a specified cable type.

The second task (Detail Design) began with a model that contained pre-defined cable interconnections, a number of which had already been routed through a sequence of cable clips to produce a harness design. It also contained three other cables that defined electrical interconnectivity but had not yet been routed to produce a physical path for these cables to follow within the harness assembly. The user was instructed to 'route the outline cables in the model through the cable clips to complete the cable harness design'. However, the individual participant needed to use their 'engineering' judgment as to what the completed harness design should be and how to achieve that goal.

The third and final task (Redesign) started with a product model that contained the design of a completed harness assembly. The participant was then given some 'engineering change requests' requiring redesign of 
the harness in some manner. The specific changes required were the addition of a new cable to the harness and the removal of one of the cables and its associated connectors from the harness.

Each task was designed to be achievable within a 20-minute immersive session with the limited application knowledge that was available to each user. Each participant attended four different sessions to complete the evaluation process; the first session being used for training. The next three sessions were used to complete the three evaluation tasks, one task per session. Ten participants completed the experiments. They were all male, eight were 20-29 years of age and two were 30-39 years of age and all had normal or corrected-to-normal vision. All were right hand dominant, with eight being right eye dominant, and two being left eye dominant. Seven of the participants estimated that they had between 10-100 hours of previous CAD experience with three estimating 100-1000 hours experience. Seven participants had no prior VR experience, two had less than 10 hours, and one had 100-1000 hours of experience.

The immersed design activity was followed by an informal discussion session during which feedback about the system and the participant's experience with it was collected. Once all the data was collected designer behavior was analyzed using two separate task categorizations, namely environmental categories and activity categories which were each split into subcategories as shown below:

Environmental Categories:

Model: anywhere within the 3D model environment;

Menu (no model visible): anywhere within the menu system without the model being visible;

Menu (with model visible): anywhere within the menu system with at least part of the model being visible; Help/Information: viewing additional textual information such as help screens or external information sources.

Activity Categories:

Design activity: the creation, editing, or deletion of objects in the environment;

Support activity: setting/viewing object parameters, system operation and viewing;

Help and other information;

Navigation/Viewpoint control;

Breaks and errors: breaks in activity between the end of one discrete action and the start of the next (for example, the time between exiting one function and entering the menu system), and all erroneous activity (such as inserting the wrong part).

The results are reported as overall averages for all participants and tasks.

Subjective measures obtained through informal interviews suggested that participants found the time immersed in the model to be of benefit, whereas the menus and help information presented in an immersive environment appeared to offer little in value over a conventional desktop CAD system. For this reason, a high percentage of time spent in the model can be perceived as a positive result (figure 20). However, when considered in the context of a maximum 20 minute spell there is certainly a need to minimize the amount of time spent on accessing menus and help. The results show that the time spent in menus could perhaps be improved by more advanced menu systems such as context sensitive menus, tool palettes and more advanced object constraints such as affordances and mating.

Figure 20. Average time distribution by environment.

Examining the average time distribution by category (figure 21) shows that a large amount of the time is spent on navigation $(23 \%)$ utilizing traditional constant speed immersive 'flying'. This is partly due to the model being presented in super-scale and points to the need to have extremely efficient forms of moving around the environment which requires further investigation, especially from a human factors point-of-view. Note that the flying speed was kept constant in this prototype system in order to reduce confounding variables in experimentation. 
Figure 21. Average time distribution by category.

A high percentage of the time spent was in erroneous activity or in breaks in activity. Since these studies were initial, investigative look at the use of a design application, advanced categorization of errors was not carried out. A major challenge in categorizing errors in a design task is that design necessarily involves iterative activity, with designs being refined many times before completion. This distinction between design iteration and erroneous activity is therefore non-trivial and requires further research. From discussions with the participants, many of the breaks seemed to be "thinking time" where the designers were thinking about the problem solution, spatial layout, the tools available to name but a few. Distinguishing between different cognitive activities is not possible with the data collected in this study; however, due to the manner in which user logging has enabled the discretization of the design task, this system now provides a useful foundation on which such studies can be built

Following on from the general categorization of the COSTAR system, more detailed analysis was performed to further understand which activities were costly in terms of time. The general activity categories were therefore decomposed into sub-categories as follows:

(1) Design was split into 'Design -goal activity', and 'Drag and Drop' activity;

(2) 'Design Support' activity was split into 'System

Operation' and 'Design Support';

(3) 'Breaks and Errors' was split into 'Inter-sequence Breaks' and

'Non Value Added Activity/Errors'.

'Navigation' and 'Help' were left as before.

The results from the experiments for these more detailed activities are given in Table 9 and figure 22 .

Table 9. Average activity by detailed category.

Figure 22. Percentage average activity by detailed category.

The categories developed and the subsequent results obtained gave a detailed breakdown of the design task, all through setting up a non-intrusive user logging procedure followed by a detailed, automated results analysis. Overall, what can be observed is that the capability to log the user in an immersive design environment in this way has provided an opportunity to carry out a powerful analysis of the design function to a level of detail that has simply not been possible in the past. The results have provided useful information relating to the most time consuming, non-productive activities being carried out and pointed to solutions for improving both the interface, menu and navigation capabilities of the system to maximize the amount of time available for engineers to carry out product engineering tasks in this crucially time limited technological environment.

\section{Conclusions}

The research highlighted in this paper demonstrates how immersive VR and the associated non-intrusive user logging can provide a powerful combination of tools for the effective analysis of product engineering activities. It also demonstrates how it is possible to automatically generate relevant engineering data using the same approach. In addition, it shows the potential for acquiring data and user contextual information in the engineering domain, pointing to a capability in the long term for the automatic acquisition of information and product development and planning knowledge for project through-life information gathering. Similar processes and procedures may be appropriate for other computer aided engineering domains, such as CAD, computer aided process planning (CAPP), production planning to name but a few. 
Due to the functional engineering nature of all of these studies the main emphasis has been on engineering tools for solving engineering problems. However, the human factors elements covered demonstrate that it is now possible for psychologists, engineers and others to use immersive VR as a tool to detail analyze complex engineering tasks in a way that was not previously possible.

\section{Acknowledgements}

The authors would like to express their thanks to the staff and personnel the industrial partners and would like to acknowledge the support of the UK Engineering and Physical Sciences Research Council and the Scottish Manufacturing Institute.

\section{References}

Clark P., Boswell R., Rule Induction with CN2: some recent improvements, in Fifth European Conference (EWSL-91), Springer-Verlag, Turing Institute, Glasgow, 1991, 151-163.

Clark P., Niblett T., The CN2 induction algorithm. Machine Learning Journal, 1989, 3, 261-283.

Conru A.B., A genetic approach to the cable harness routing problem, in IEEE Conference on Evolutionary Computation, 1994, 1, $200-205$.

Conru A.B., Cutkosky M.R., Computational support for interactive cable harness routing and design, in 19th Annual ASME Design Automation

Conference, 1993, 551-558.

Cruz-Niera C., Making virtual reality useful: a report on immersive applications at Iowa State University, in Future Generation Computer Systems,

1998, 14, 147-155.

Dani T.H., Gadh R., Creation of concept shape design via a virtual reality interface. Journal of Computer Aided Design, $1997,29,555-563$.

Dewar R.G., Assembly plans from virtual environments. PhD Thesis, Heriot-Watt University, UK, 1998.

Dewar R.G., Carpenter I.D., Ritchie J.M., Simmons J.E.L. Assembly planning in a virtual environment, in PICMET '97, Portland, USA, 1997a,

664-667.

Dewar R.G., Ritchie J.M., Carpenter I.D., Simmons J.E.L., Tools for assembly in a virtual environment, in ICMA '97, University of Hong Kong,

1997b, 1, 583-587.

Digital Art Forms Ltd.: Available online at: http://www.digitalartforms.com/home.htm, (accessed 16 March, 2006).

Fischer A.G., Chipperfield K., Vance J.M., VR Hose: hydraulic hose routing in virtual reality with Jack ${ }^{\mathrm{TM}}$. In Expert Systems, edited by R.

Forsyth, 2nd Edition, 2002 (Chapman and Hall Computing).

Gomes de Sa A., Zachmann G., Virtual reality as a tool for verification of assembly and maintenance processes. Computers and Graphics Journal,

1999, 23, 389-403.

Gupta R., Prototyping and design for assembly analysis using multimodal virtual environments. PhD Thesis, Massachusetts Institute of

Technology, USA, 1995.

Hart A., Knowledge Acquisition for Expert Systems. 1996 (Kogan Page).

Hayes N., Doing Psychological Research, 2000 (Open University Press).

Heger R., Richter M., Advanced assembly planning using virtual reality techniques, in European Conference on Integration in Manufacturing,

1997, 73-82.

Howarth P.A., The Multi-Factorial Causes of Virtual Simulation Sickness. VISERG Report, HSE Contract Research Report 274/2000, 1997.

Jayaram S., Connacher H.I., Lyons K.W., Virtual assembly using virtual reality techniques. Computer-Aided Design Journal, $1997,29,575-584$.

Jayaram, S., Vance, J., Gadh, R., Jayaram, U., Srinivasan, H., Assessment of VR technology and its application to engineering problems. Journal

of Computing and Information Science in Engineering, 2001, 1(1), 72-83.

Kay F., Constantin C., An IT view on perspectives of computer aided process planning research. Computers In Industry Journal, 1997, 34,

307-337.

Kelly G.A., The Psychology of Personal Constructs, 1995 (Norton).

Ng F.M, Ritchie J.M., Simmons J.E.L., Dewar R.G., Designing cable harness assemblies in virtual environments. Journal of Materials Processing

Technology, 2000, 107, 37-43.

Nonaka I., Takeuchi H., The Knowledge-Creating Company, 1995 (Oxford University Press).

Preece J., Rogers Y., Sharp H., Benyon D., Holland S., Carey T., Human-Computer Interaction, 1994, (Addison-Wesley).

Ritchie, J.M., The present and future role of virtual reality in the "Engineering a Product" cycle, in 5th International Conference on Integrated

Design and Manufacturing in Mechanical Engineering (IDMME'04), University of Bath, Bath, UK, 2004.

Weyrich M., Drews P., An interactive environment for virtual manufacturing: the virtual workbench. Computers in Industry Journal, 1999, 38, 
5-15.

Wolter J., Kroll E., Towards assembly sequence planning with flexible parts, in IEEE International Conference on Robotics and Automation, 1996, 1517-1524.

Zhu D., Latombe J.C., Pipe routing = path planning (with many constraints), in IEEE International Conference on Robotics and Automation, 1991, 1940-1947. 